\title{
HARMONIC EXCITATION OF MANTLE RAYLEIGH WAVES BY THE 1991 ERUPTION OF MOUNT PINATUBO, PHIIIIPPINES
}

\author{
Hiroo Kanamori \\ Seismological Laboratory, California Institute of Technology \\ Jim Mori
}

U. S. Geological Survey

\begin{abstract}
An unusually long (at least two hours) seismic wave train having periods of about $230 \mathrm{sec}$ was recorded at many worldwide seismic stations during the major eruption of Mount Pinatubo in the Philippines on June 15, 1991. This wave train exhibits two sharp spectral peaks at 228 and 270 sec. The group velocity, phase velocity, and the particle motion of this wave train indicate that it is a Rayleigh wave. The most probable excitation mechanism is acoustic coupling of atmospheric oscillations that were set off by continuous thermal energy flux from the volcano. The two spectral peaks correspond to the characteristic periods of acoustic and gravity modes of the Earth's atmosphere. The magnitude of the vertical single force equivalent to the acoustic coupling is $1.6 \times 10^{17}$ dynes over a frequency band of 0.003 to $0.01 \mathrm{~Hz}$. The results suggest the possibility of using acoustically coupled Rayleigh waves for detection, characterization and quantification of volcanic eruptions. Acoustic coupling of the atmosphere and the solid Earth provides a unique seismic source with long duration.
\end{abstract}

\section{Introduction}

An unusually long (at least two hours) seismic wave train having periods of about $230 \mathrm{sec}$ was recorded during the major eruption of Mount Pinatubo in the Philippines on June 15,1991 . This wave train was observed consistently at many broadband seismic stations throughout the world including Japanese, TERRAscope (Caltech's broadband array), IRIS (Incorporated Research Institutions for Seismology) and IDA (International Deployment of Accelerometers)/IRIS networks. It was also recorded on a gravimeter at the Black Forest Observatory, Germany, and was identified as the oscillation originated from the Pinatubo eruption (Walter Zürn, written communication, 1991, Widmer and Zürn, 1992).

Since this kind of wave train has never been observed, we document the observed records, and show that the wave train is a Rayleigh wave excited by atmospheric oscillations near the volcano that were set off by continuous thermal energy flux from the volcano.

\section{Observation}

Figure 1 shows the three-component very-long-period (VLP) seismograms recorded at MAJO (Matsushiro, Japan) during the Pinatubo eruption, where the unusual long-period

Copyright 1992 by the American Geophysical Union.

Paper number 92GL00258

0094-8534/92/92GL-00258\$03.00

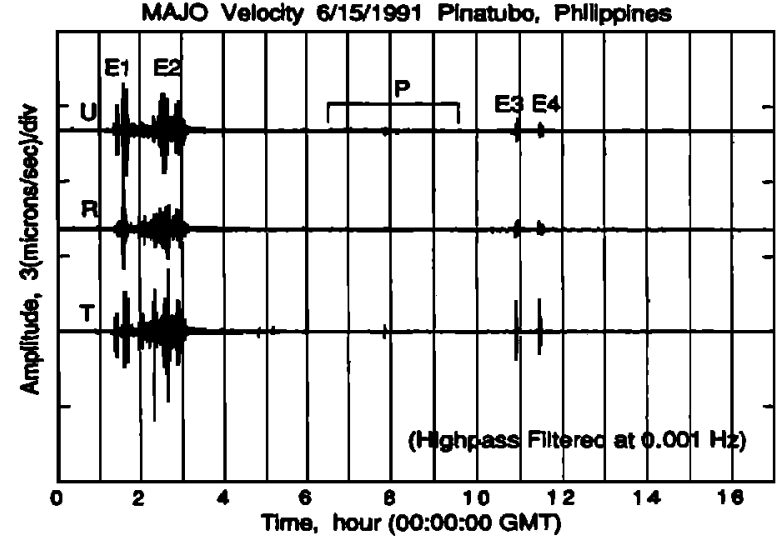

Fig. 1. The three-component (vertical and two horizontal components rotated in radial and transverse directions with respect to the Philippines) very-long-period (VLP) seismograms recorded at MAJO $\left(\Delta=26.4^{\circ}\right)$. E1 and E2 indicate earthquakes in the Caucasus $(M=6.3)$ and the South Sandwich Islands $(M=6.5)$, respectively. E3 and E4 indicate two earthquakes ( $M=5.5$ and 5.3, respectively) in the Philippines associated with the Pinatubo eruption. The longperiod wave train excited by the Pinatubo eruption is indicated by $P$.

wave train is indicated by P. Symbols E1 and E2 indicate earthquakes in the Caucasus $(M=6.3)$, and the South Sandwich Islands ( $M=6.5)$, respectively. Symbols $E_{3}$ and $E_{4}$ indicate two earthquakes ( $M=5.5$ and 5.3, respectively) in the Philippines associated with the Pinatubo eruption. The wave train labelled $\mathrm{P}$ is almost harmonic as shown by Figure 2, which shows the spectrum of the first half of the wave rain

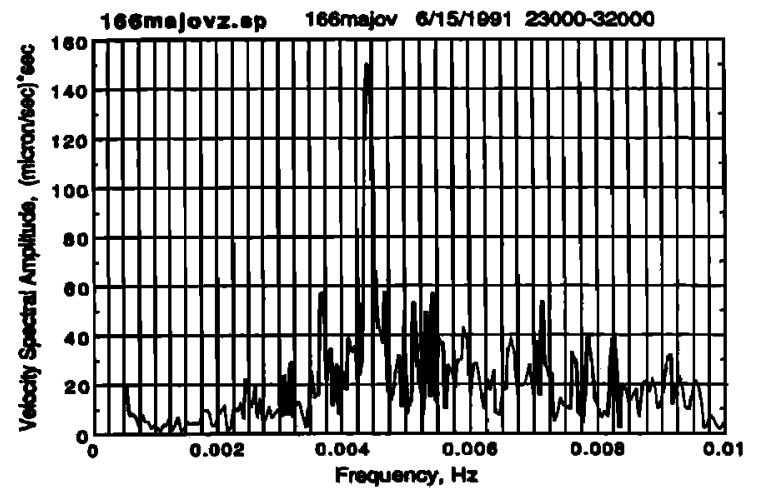

Fig. 2. The spectrum of the vertical component of the MAJO record shown in Figure 1. The time window used is from 6:23 to 8:53 GMT. 
(from 06:23 to 08:53 GMT). The spectrum indicates a dominant peak at $228 \mathrm{sec}$, and another minor peak at about $270 \mathrm{sec}$.

Figure 3 shows bandpass $(0.0033$ to $0.005 \mathrm{~Hz})$ filtered records of the wave train recorded at stations with distances ranging from $22^{\circ}$ to $132^{\circ}$, and a $230^{\circ}$ spread of azimuth. The time axis refers to the nearest station, SHK (Shiraki, Japan), and the other traces are time-shifted by the respective group travel times assuming a velocity of $3.55 \mathrm{~km} / \mathrm{sec}$. The wave train labelled $R_{1}$, which is very coherent from station to station, has a group velocity of $3.55 \mathrm{~km} / \mathrm{sec}$ corresponding to the group velocity of Rayleigh waves at a period of $228 \mathrm{sec}$. This indicates that this wave train is a Rayleigh wave. Also, as shown in Figure 4, the amplinude ratio and the phase shift between the vertical and radial components recorded at MAJO (Matsushiro, Japan) are consistent with those of Rayleigh waves, and the transverse component is much smaller than the vertical or radial component.

The duration of this wave train is at least two hours. Figures 1 and 3 suggest that it could be as long as six hours, but the latter half of this wave train is obscured by possible interference of $\mathbf{R}_{\mathbf{2}}-, \mathbf{R}_{\mathbf{3}}$-wave trains and ground motions caused by the passage of air waves $\left(A_{1}\right)$, as illustrated on Figure 3 . The phase relation between the vertical and radial components for the latter half varies with time, suggesting complex interference. For example, at MAJO and TSK which are only $1^{\circ}$ apart, the interference pattern between $R_{2}$ and $R_{3}$ is very different.

For a travelling wave with a wave length, $\lambda$, destructive and constructive interference between $R_{2}$ and $R_{3}$ alternate at points $\lambda / 4$ apart. Since the wave length of the Rayleigh wave shown in Figure 3 is about $1000 \mathrm{~km}$, the drastic difference in the interference pattern between MAJO and TSK is expected.

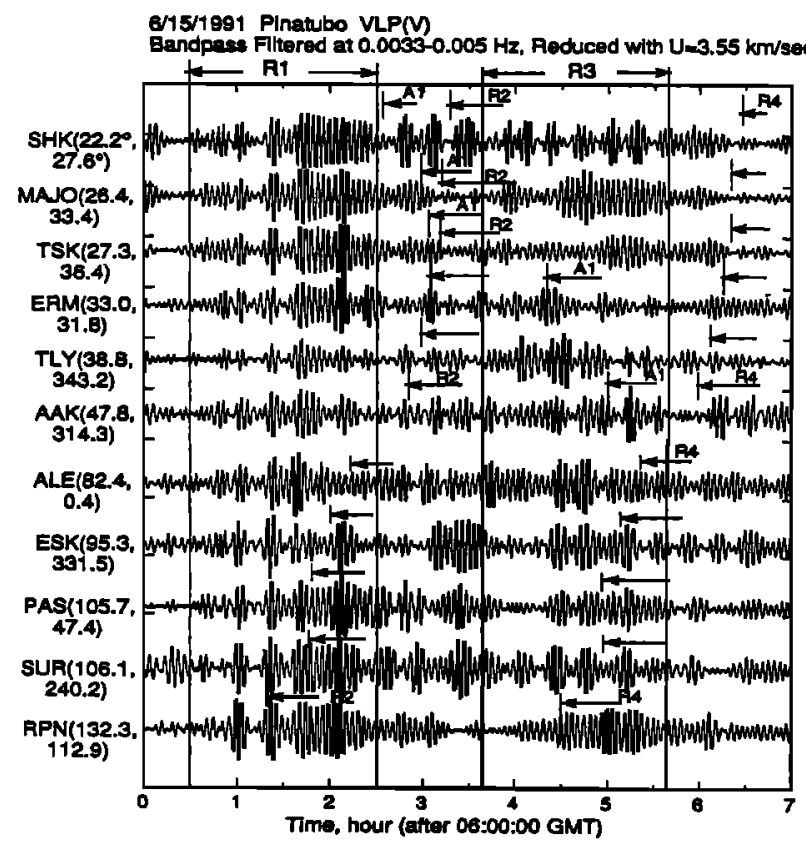

Fig. 3. Bandpass filtered seismograms recorded at global broadband stations. The beginning of the trace for SHK is at 6:00 GMT on June 15, 1991. The traces for other stations are time-shifted by the respective group travel times computed with a velocity of $3.55 \mathrm{~km} / \mathrm{sec}$. The first number in the parenthesis after the station name is the distance and the second is the azimuth. $R_{1}, R_{2}, R_{3}$, and $A_{1}$ indicate times of successive Rayleigh waves and the direct air wave, respectively.

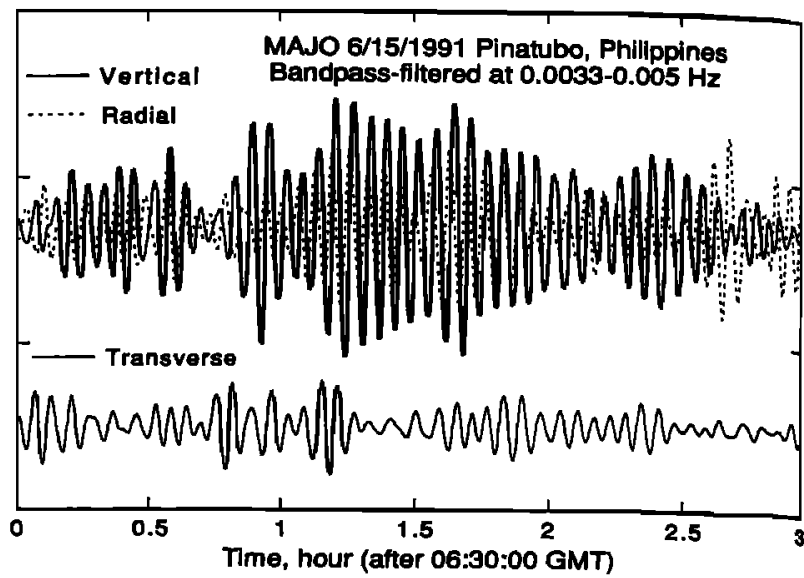

Fig. 4. The vertical, radial, and transverse components of the wave train recorded at MAJO. The vertical and the radial components are superposed to show the prolate retrograde particle motion of Rayleigh waves coming from the Philippines.

Figure 5 shows the phase arrival time of the $R_{1}$-wave train reduced with a velocity of $4.791 \mathrm{~km} / \mathrm{sec}$, which is the phase velocity of Rayleigh waves at a period of $228 \mathrm{sec}$. The deviation from the zero line shows the sum of the source phase and the residual propagation phase delay along the path. The deviation is less than $40 \mathrm{sec}$, which is about the right magnitude for regional variations of the phase velocity. Thus we conclude that the source phase is essentialiy independent of azimuth.

Figure 6 compares the barogram recorded at the Clark Air Force base, $21 \mathrm{~km}$ from Mount Pinatubo, with the seismic signal at MAJO, plotted with a common time scale. The maximum pressure change recorded on the barogram is about 3.5 mbars.

\section{Interpretation}

We suggest two possibilities for the excitation mechanism of this long Rayleigh-wave train. The first mechanism involves some periodicity in the eruption process itself. Since the period is long compared with any conceivable acoustic resonance periods for the magma chamber, the mechanisms that are often used to explain volcanic tremor (e.g. Aki and Koyanagi, 1981) are not appropriate. However, if some thermal process such as cooling and hardening of magma is involved, it is possible

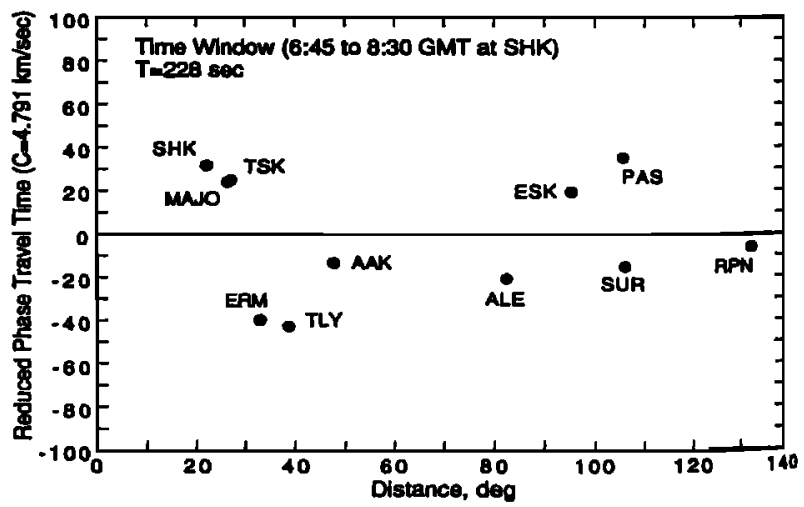

Fig. 5. The phase arrival times of the $R_{1}$-wave train at $\mathrm{T}=228 \mathrm{sec}$ reduced with a phase velocity of $4.791 \mathrm{~km} / \mathrm{sec}$. 


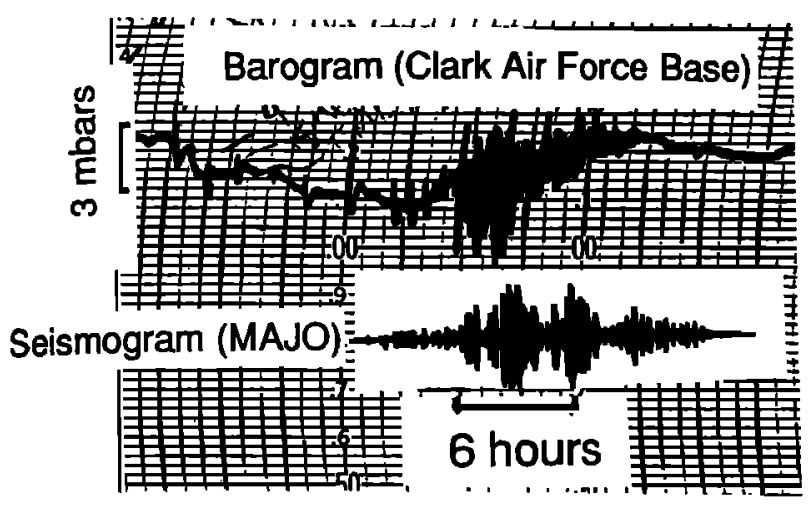

Fig. 6. The pressure change observed at the Clark Air Force Base $(\Delta=21 \mathrm{~km}$ from Mount Pinatubo). The seismogram recorded at MAJO is shown with a common time scale.

for a volcanic process to have a very long periodicity. The several-hour periodicity of geyser eruptions is an example. However, no such mechanism is known for volcanic eruptions.

The second mechanism involves excitation of the atmosphere. The eruption of Mount Pinatubo was a long continuous process which transferred a large amount of thermal energy from the Earth's interior into the air. The earth's atmosphere has two distinct velocity minima, one at a height of about $20 \mathrm{~km}$, and the other at $80 \mathrm{~km}$. The thermal perturbation can continuously excite oscillations of the stratified atmosphere. The oscillation of the atmosphere by volcanic eruptions and atmospheric nuclear explosions has
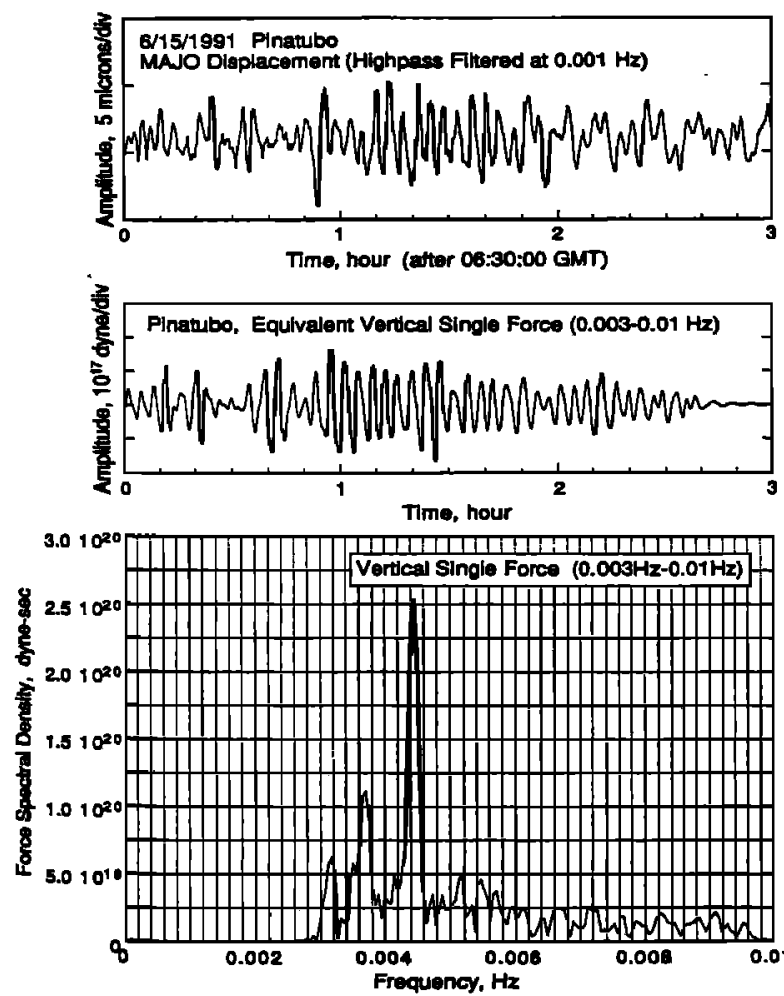

Fig. 7. Top: Displacement record at MAJO used for deconvolution. Middle: The derived time history of the vertical single force at the source. Bottom: The spectrum of the vertical single force. been studied extensively by many investigators. In general, two modes of oscillation, acoustic and gravity modes, exist. Since the atmosphere is not confined laterally, the energy excited by the source propagates away laterally and is observed as dispersive acoustic-gravity waves in the farfield. Harkrider (1964), Press and Harkrider (1962), and Pfeffer and Zarichny (1963) computed dispersion curves of air waves for many models of atmospheric columns. Their results show two group velocity minima at periods of about $250 \mathrm{sec}$, which can be interpreted as the periods of the acoustic and gravity modes of the earth's atmosphere. Although no nearfield waveforms have been computed by these authors, we expect near-vertical oscillations of the atmosphere near these periods. The proximity of these periods to those of the two observed spectral peaks suggests that acoustic coupling of atmospheric oscillations to the ground is responsible for excitation of the observed Rayleigh waves. Since the exact periods depend on the local air temperature and density, the difference between the observed and computed periods is not significant.

A satellite image of Mt. Pinatubo taken by the National Oceanic and Atmospheric Administration (NOAA) at 07:30 GMT on June 15 indicates a temperature perturbation associated with the eruption extending over an area 300 to $500 \mathrm{~km}$ from the summit. A clear wave-like structure with a wavelength of approximately $70 \mathrm{~km}$ near the summit is observed. If the sound velocity is $0.3 \mathrm{~km} / \mathrm{sec}$, the corresponding period is about $230 \mathrm{sec}$. This observation supports the above interpretation.

We explore the second possibility further by modeling atmospheric excitation with the application of a vertical single force at the epicenter. The azimuthally independent source phase justifies the use of the vertical force. We computed Rayleigh waves, $g(t)$, excited by a delta function single force by summation of normal modes using equation (3) of Kanamori and Given (1982). Then we obtained the force time history, $f(t)$, by deconvolving the observed record, $h(t)$, with $g(t)$. Figure 7 shows $h(t), f(t)$ and the spectrum of $f(t)$. For this computation we used the record obtained at MAJO which is the closest station with good calibration. The amplitude of $f(t)$ is approximately $1.6 \times 10^{17}$ dynes using the data over a frequency band from 0.003 to $0.01 \mathrm{~Hz}$. Since the waveform is very similar at all the stations, use of the other records would yield a similar source-time function.

This force is approximately $1 / 30$ and $1 / 3$ of the force estimated for the landslide and the explosion, respectively, associated with the 1980 Mount St. Helens eruption (Kanamori et al. 1984). The smaller amplitude of the force reflects the difference in eruptive styles between Mount Pinatubo and Mount St. Helens. The Mount St. Helens eruption began with a very strong initial explosion, exerting a large sudden force on the Earth. In contrast, the activity at Mount Pinatubo built up gradually with smaller explosions and continuous ash emissions for several hours leading up to the climactic eruption. Although the force is smaller at Mount Pinatubo compared to Mount St. Helens, the total seismic energy is much larger because of the long duration of the eruption.

Since the farfield phase velocity of air waves is much slower than the Rayleigh-wave phase velocity, acoustic coupling is generally inefficient in the farfield. However, near the source, the oscillation is near-vertical with an infinitely large phase velocity. Within a short distance from the source where the phase velocity is comparable to, or larger than, that of the seismic Rayleigh waves, efficient acoustic energy coupling is expected. 
The magnitude of the force is consistent with the amplitude of the pressure changes, 3.5 mbars, observed at Clark Air Force Base (Figure 6). If the pressure change $p$ occurred simultaneously over a circular area with radius $a$, the total force is $f=\pi p a^{2}$. To produce the estimated magnitude of the force, $1.6 \times 10^{17}$ dyne, $a$ reeds to be $37 \mathrm{~km}, 1.8$ times the distance of Clark Air Force Base from Mount Pinatubo. This dimension is also similar to the $70 \mathrm{~km}$ wavelength observed in the satellite image.

\section{Conclusions}

We showed that the observed long wavetrain is a Rayleigh wave excited by atmoșpheric oscillations set off by the eruption of Mount Pinatubo. If the excitation mechanism considered above is correct, we would expect similar oscillations for other eruptions: Eissler (1986) reported Rayleigh waves excited by the 1982 eruption of El Chichón in Mexico. Although the Rayleigh waves reported by Eissler exhibit no obvious periodicity, the excitation mechanism could be similar.

It is possible that the atmospheric pressure changes could have modulated the thermal energy flux coming out from the volcano causing seismic excitation from the interior of the earth, but this mechanism is considered unlikely.

The importance of this study is twofold. First, we demonstrated that, with the advent of high-quality global seismic networks, we can estimate the strength of acoustic coupling of the atmosphere to the solid Earth. This suggests the possibility of using acoustically coupled Rayleigh waves for detection, characterization and quantification of major volcanic eruptions. Second, acoustic coupling of the atmosphere provides a unique long-period seismic source with a long duration.

Acknowledgements. During the course of this study, we greatly benefitted from discussions with Don Anderson, Tom Heaton, Andy Ingersoll, and David Harkrider. The seismograms used in this study were made available from the stations of IRIS, TERRAscope, the Earthquake Research Institute of the University of Tokyo (by courtesy of Kiyoshi Takano), and IDA/IRIS (by courtesy of the University of
California, San Diego). Satellite imagery was provided by George Stephens and Mike Matson of NOAA. This research was partially supported by a grant from the L. K. Whittier Foundation. Contribution No. 5105, Division of Geological and Planetary Sciences, California Institute of Technology, Pasadena, California.

\section{References}

Aki, K., and R. Koyanagi, Deep volcanic tremor and magma ascent mechanism under Kilauea, Hawaii, J. Geophys. Res., 86, 7095-7109,1981.

Eissler, H. K., Investigations of earthquakes and other seismic sources in regions of volcanism, thesis, $174 \mathrm{pp}$., California Institute of Technology, Pasadena, 1986.

Harkrider, D. G., Theoretical and observed acoustic-gravity waves from explosive sources in the atmosphere, $J$. Geophys. Res., 69, 5295-5321,1964.

Kanamori, H., and J. W. Given, Analysis of long-period seismic waves excited by the May 18, 1980, eruption of Mount St. Helens -- a terrestrial monopole?, J. Geophys. Res., 87, 5422-5432, 1982.

Kanamori, H., J. W. Given, and T. Lay, Analysis of seismic body waves excited by the Mount. St. Helens eruption of May 18, 1980, J. Geophys. Res., 89, 1856-1866, 1984.

Pfeffer, R. L., and J. Zarichny, Acoustic gravity wave propagation in an atmosphere with two sound channels, Geofis. Pura. Appl., 55, 175-199, 1963.

Press, F., and D. Harkrider, Propagation of acoustic-gravity waves in the atmosphere, J. Geophys. Res., 67, 3889$3908,1962$.

Widmer, R. and W. Zürn, "Screaming" volcanoes vs. "Silent" earthquakes, Geophys. Res. Lett. submitted, 1992.

H. Kanamori, Seismological Laboratory, California

Institute of Technology, Pasadena, CA 91125

Jim Mori, U. S. Geological Survey, Pasadena, CA 91106

(Received December 26, 1991; accepted January 31, 1992.) 